\title{
Time-resolved analysis of the precursor fragmentation kinetics in an hybrid PVD/PECVD dusty plasma with pulsed injection of HMDSO
}

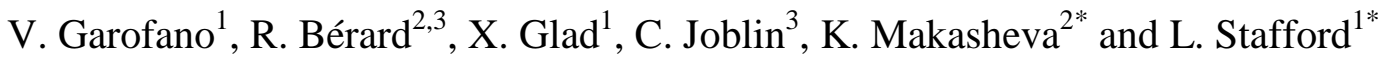 \\ ${ }^{1}$ Département de physique, Université de Montréal, Montréal, Québec, Canada \\ ${ }^{2}$ LAPLACE (Laboratoire Plasma et Conversion d'Energie), Université de Toulouse, CNRS, UPS, INPT, Toulouse, France \\ ${ }^{3}$ IRAP (Institut de Recherche en Astrophysique et Planétologie), Université de Toulouse, CNRS, UPS, CNES, Toulouse, France
}

\begin{abstract}
Plasma sampling mass spectrometry (PSMS) has been carried out to study the fragmentation kinetics of hexamethyldisiloxane (HMDSO) in a low-pressure, axially asymmetric argon rf discharge designed for the growth of nanocomposite thin films through an hybrid PVD/PECVD method. Experiments have been conducted with pulsed injection of HMDSO over a 5-s period. Plasma conditions have been chosen to favor formation and disappearance of dust occurring in cycles of a few hundred seconds. The dissociation degree of HMDSO and the relative intensities of HMDSO-related fragments are reported and analyzed regarding these two specific timescales. PSMS showed that formation of dust increases HMDSO dissociation. The same result can be deduced from the particle balance equation of HMDSO using the electron density and temperature obtained from optical emission spectroscopy as the only input parameters. For HMDSO, electron-impact dissociation is the dominant loss pathway over diffusion and recombination on the reactor walls. Small $\mathrm{C}_{\mathrm{x}} \mathrm{H}_{\mathrm{y}}$ compounds and $\mathrm{H}_{2}$ are mostly generated from surface recombination mechanisms and lost by electron-impact dissociation.
\end{abstract}

\footnotetext{
*Corresponding authors: kremena.makasheva@laplace.univ-tlse.fr; luc.stafford@umontreal.ca
} 


\section{Introduction}

Hexamethyldisiloxane (HMDSO, $\mathrm{Si}_{2} \mathrm{O}\left(\mathrm{CH}_{3}\right)_{6}$ ) is a well-known precursor used in plasmaenhanced chemical vapor deposition (PECVD) processes to introduce the silicon element in the plasma and thus in the coating. Hence, it relates to numerous applications ${ }^{1-6}$ such as the production of hard coatings ${ }^{7}$, superhydrophobic surfaces ${ }^{8-10}$, multifunctional nanocomposites thin films ${ }^{11-14}$ and several other industrially relevant materials and nanomaterials ${ }^{15-17}$. HMDSO is also widely used for its convenience regarding the growth of polymer films because of its relatively low-cost and non-toxicity during manipulation. Introduced as a precursor, the plasma dissociates the molecule into fragments which then interact with the substrate to form a film, with initial plasma conditions (pressure, power, wall cleaning, etc.) strongly influencing the structural, electrical and optical properties of the final coating. Thus, numerous studies aimed at optimizing the growth rate and the general quality of the film by changing the plasma conditions such as gas mixture, pressure and applied power ${ }^{18-20}$.

Apart from the characterization of the coatings produced with HMDSO by PECVD, a lot of work has been done to study the gas phase properties in order to better understand and control the plasma process. Optical emission spectroscopy (OES) has been carried out to identify the complex emission signature of HMDSO-containing plasmas ${ }^{21-24}$. Various optical absorption spectroscopy techniques were also used to assess the variations of the gas phase composition with changes of the experimental conditions ${ }^{21-23}$. Plasma sampling mass spectrometry (PSMS) has further been used to detail the dissociation path of HMDSO that leads to the formation of its principal fragments ${ }^{25-34}$. However, the interpretation of the recorded data is not trivial and a deep understanding of the dynamics involved in such processes is needed. Most noticeably, Basner et al. derived ionization cross sections of HMDSO and its dominant compounds ${ }^{25}$ while Alexander 
et al. correlated signals to possible species in the plasma to propose fragmentation and recombination paths that would lead to oligomerization ${ }^{26,27}$. Yet, controlling the number densities of the desired fragments remains a challenge. Indeed, plasma species, such as ions, metastable atoms, photons and electrons, are all likely to play a significant role in the fragmentation of HMDSO; a fine tuning would require specific conditions where density and energy of said species are known and controlled.

Extensive work has been carried out to understand the effect of the power density and gas composition on the fragmentation kinetics of $\mathrm{HMDSO}^{27}$. Nonetheless, time-resolved plasma sampling mass spectrometry (PSMS) measurements in dynamic plasma conditions i.e., where such parameters significantly vary in time, were rarely reported even though plasma deposition processes are often operated in such conditions. One could think for example of pulsed plasmas where the injected power is applied with a certain frequency and duty cycle. The injection of the precursor in the gas mixture can also be pulsed when target poisoning becomes an issue, such as hybrid PVD/PECVD processes ${ }^{12-14}$. In this case, the target, usually metallic, is sputtered by highenergy ions to obtain metal nanoparticles while the precursor, leading to formation of a matrix to embed the nanoparticles in a deposit on the substrate, is also injected in the gas phase ${ }^{12,13,35}$. Such technique allows for the growth of nanocomposite thin films, where different phases are present throughout the depth of the film. Though judicious control of the operating conditions, it was however found that pulsing the injection of HMDSO can lead to the formation of successive generations of nanoparticles, or dust, in the gas phase ${ }^{36}$. A recent PSMS study in such conditions showed that acetylene $\left(\mathrm{C}_{2} \mathrm{H}_{2}\right)$ is formed as a result of HMDSO fragmentation in Ar/HMDSO plasmas $^{37}$ and may be considered as the main origin of dust formation in analogy with hydrocarbon dusty plasmas. Acetylene-based dusty plasmas have been extensively studied ${ }^{38-42}$, 
proving that $\mathrm{C}_{2} \mathrm{H}_{2}$ is a strong dust promoter via the confinement of the $\mathrm{C}_{2} \mathrm{H}^{-}$ion. The formation of nanoparticles in such plasmas requires consumption of the same compounds as the available HMDSO-related fragments in Ar/HMDSO plasmas. Therefore, a hypothesis based on $\mathrm{C}_{2} \mathrm{H}_{2}$ for dust formation in Ar/HMDSO plasmas, like in hydrocarbon plasmas, is realistic. It however remains unclear for HMDSO-containing plasmas which fragments are the most affected by the dust growth and to which extent.

In a recent study, a multi-scale evolution of the electron temperature and electron number density in a low-pressure, axially asymmetric argon rf plasma with pulsed injection of HMDSO was obtained by OES of Ar 2p-to-1s transitions coupled with a collisional-radiative model describing the populations of emitting $\operatorname{Ar} 2 p \operatorname{states}^{43}$. The results have shown that the injection of HMDSO reduces the electron mean energy since (i) the HMDSO molecule and its fragments decrease the mean ionization threshold of the nominally pure argon plasma and (ii) enables Penning ionization through collisions between HMDSO-related species and argon metastable atoms. The opposite trend was observed for the electron density. A rise of the electron temperature and a decrease of the electron density are typically observed in presence of a dust cloud.

In the present work, time-resolved OES measurements are recorded simultaneously with time-resolved PSMS measurements aiming to establish a link between the evolution of the fragmentation of the precursor and the electron temperature and electron density throughout the experiment. The dynamics taking place here occur at two different time-scales: the pulsed injection of HMDSO having a 5-s period and the successive formation and disappearance of the dust cloud occurring over cycles of a few hundred seconds. This distinctive feature allows us to separate the two phenomena while studying them simultaneously. Thus, we can analyze both the 
impacts of the HMDSO injection and of the dust formation on the precursor fragmentation kinetics, and more generally on the plasma chemistry.

\section{Experimental setup and diagnostics}

The plasma setup used in this study has been designed for the growth of nanocomposite thin films ${ }^{12}$ using a hybrid PVD/PECVD method. PVD is achieved by sputtering of a metal target (silver in the present case) with argon ions at low pressure while PECVD is carried out by injecting a precursor in the gas mixture: HMDSO in the current experiment. The experimental setup is depicted in Figure 1. The plasma is generated in an axially-asymmetric capacitivelycoupled radiofrequency (rf) discharge. The 13.56-MHz rf power is applied on the top, smaller electrode, which consists of a silver target with a diameter of $10 \mathrm{~cm}$. The bottom, larger electrode (diameter of $12 \mathrm{~cm}$ ), which serves as the substrate holder when deposition of thin films is performed, is grounded, as well as the chamber walls. This asymmetry in the reactor geometry allows for a self-bias voltage on the powered electrode that can be increased to more than a thousand of volts at the target surface depending on the applied power. The self-bias voltage is at the origin of the acceleration of positive ions towards the smaller electrode, thus enabling the sputtering of the target. The residual pressure in the plasma reactor, obtained with a turbomolecular pump, is of $2 \times 10^{-6}$ Torr. The argon base pressure for operation in the current study is of 40 mTorr (corresponding to an Ar-flow of $2.8 \mathrm{sccm}$ ). The injection of the HMDSO precursor is pulsed at $0.2 \mathrm{~Hz}$. When the injection time on $\left(\mathrm{t}_{\mathrm{on}}\right)$ is varied, the injection time off $\left(t_{\text {off }}\right)$ is adjusted such that the period equals $5 \mathrm{~s}\left(\mathrm{~T}=\mathrm{t}_{\mathrm{on}}+\mathrm{t}_{\mathrm{off}}=5 \mathrm{~s}\right)$. The gas mixture is introduced in the discharge through a periodically pierced ring surrounding the top electrode. The maximum available HMDSO flow is of $0.4 \mathrm{sccm}$, corresponding to a continuous injection of HMDSO, 
which represents only a 4 mTorr of partial pressure. Note that the introduction of HMDSO in the gas phase induces deposition and consequently partial or complete covering of the target with organosilicon compounds. The HMDSO flow is adjusted by a mass flow controller OMICRON switched by a pulse generator AGILENT. It was observed that, under particular conditions, namely an applied power below $60 \mathrm{~W}$ and an HMDSO duty cycle above $30 \%$, the pulsed injection of the precursor induces periodic growth of dust, forming a cloud of nanoparticles between the two electrodes ${ }^{36,37,43}$.

The HAL EQP 1000 mass spectrometer provided by Hiden Analytical is set perpendicularly to the axis of the electrodes, so that the entrance of the mass spectrometer is close to the level of the chamber walls (as in Despax et $a l .{ }^{37}$ ). It operates here in residual gas analysis (RGA) mode to probe the population of neutral species in the plasma. In the ionization chamber, at the entry of the PSMS, the energy of the electrons coming from the filament is set at $20 \mathrm{eV}$. This value was chosen since it is above the ionization energy of argon $(15.76 \mathrm{eV})$ and HMDSO $(8.8 \mathrm{eV})$ as well as the threshold energy required for dissociative ionization of the HMDSO fragments $\left(\mathrm{Si}_{2} \mathrm{O}\left(\mathrm{CH}_{3}\right)_{5}: 9.8 \mathrm{eV}, \mathrm{CH}_{4}: 12.6 \mathrm{eV}, \mathrm{C}_{2} \mathrm{H}_{2}: 11.4 \mathrm{eV}, \mathrm{Si}_{2} \mathrm{OH}\left(\mathrm{CH}_{3}\right)_{4}\right.$ and $\left.\mathrm{Si}\left(\mathrm{CH}_{3}\right)_{3}: \sim 18 \mathrm{eV}\right)^{25,30}$. The residual pressure in the PSMS is $7 \times 10^{-8}$ Torr and goes up to $6 \times 10^{-}$ ${ }^{7}$ Torr during plasma operation. Additionally, an optical fiber is mounted in front of a quartz window at the discharge inter-electrode gap and connected to an AvaSpec 3648 optical spectrometer provided by Avantes Inc. ranging between 700 and $900 \mathrm{~nm}$ with a $0.05-\mathrm{nm}$ resolution (full width at half maximum) to perform OES measurements. 
(a) side view

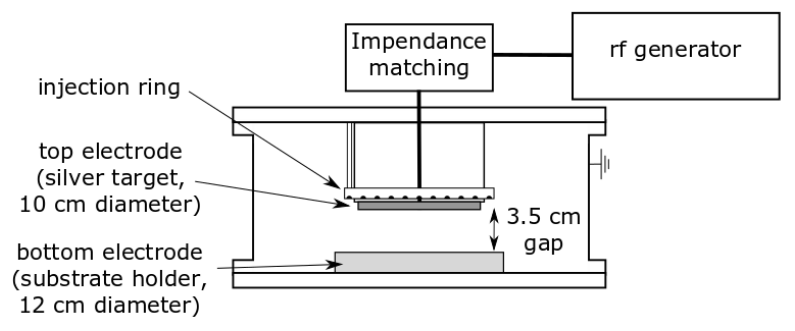

(b) top view

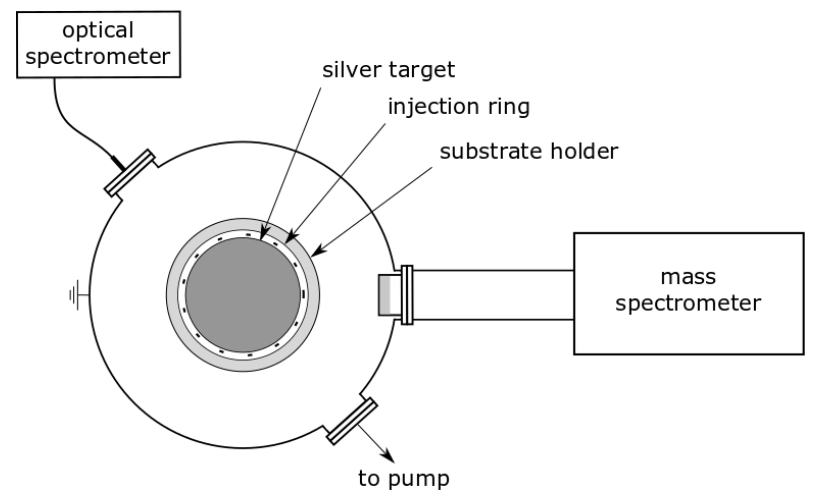

Figure 1: Side (a) and top (b) views of the experimental setup comprising the reactor and diagnostics used throughout this study.

\section{Plasma sampling mass spectrometry measurements}

Mass spectra for the Ar/HMDSO gas mixture at plasma off and on states are presented in Figure 2. Since the injection of the precursor is pulsed with a frequency of $0.2 \mathrm{~Hz}$ and that each scan takes a few tens of seconds, all spectra presented in this work are averaged over 3 scans to limit temporal variations. Argon atoms appear as the dominant species, as expected from the values of the different partial pressures. Also, significant signals observed at $\mathrm{m} / \mathrm{z}=18$ a.m.u. $\left(\mathrm{H}_{2} \mathrm{O}\right.$, which thereafter will be simply expressed as $\left.\mathrm{m} / \mathrm{z} 18\right)$, and $\mathrm{m} / \mathrm{z} 28\left(\mathrm{~N}_{2}\right)$ are due to the presence of humidity in the PSMS and impurities in the reactor.

The mass spectrum at plasma-off state shows the cracking pattern of HMDSO $\left(\mathrm{Si}_{2} \mathrm{O}\left(\mathrm{CH}_{3}\right)_{6}\right)$. Although a signal is seen at $\mathrm{m} / \mathrm{z} 162$, corresponding to $\mathrm{Si}_{2} \mathrm{O}\left(\mathrm{CH}_{3}\right)_{6}{ }^{+}$, the dominant 
signal attributed to HMDSO is at $\mathrm{m} / \mathrm{z} 147$, corresponding to the parent molecule minus a methyl group, $\mathrm{Si}_{2} \mathrm{O}\left(\mathrm{CH}_{3}\right)_{5}{ }^{+}$or HMDSO-15. This feature was already observed in previous mass spectrometry investigations of HMDSO-containing plasmas and is explained by the fact that energized HMDSO can easily dissociate with $\mathrm{CH}_{3}$; the $\mathrm{CH}_{3}$-loss being the lowest fragmentation channel $^{25,26,30,37,44}$. Significant signals related to the HMDSO fragmentation are also observed at other masses. They are reported in Table 1, along with their corresponding fragment.

Table 1 : List of the signals observed by PSMS at plasma-off that correspond to the fragmentation of HMDSO.

\begin{tabular}{cc}
\hline $\begin{array}{c}\text { Signal } \\
(\mathbf{m} / \mathbf{z})\end{array}$ & Ion $^{25}$ \\
\hline 45 & $\mathrm{SiCH}_{5}^{+}$ \\
57 & $\mathrm{SiC}_{2} \mathrm{H}_{5}^{+}$ \\
73 & $\mathrm{Si}^{+}\left(\mathrm{CH}_{3}\right)_{3}{ }^{+}$ \\
131 & $\mathrm{Si}_{2} \mathrm{OC}_{4} \mathrm{H}_{11}{ }^{+}$ \\
147 & $\mathrm{Si}_{2} \mathrm{O}\left(\mathrm{CH}_{3}\right)_{5}^{+}$
\end{tabular}

After plasma ignition (Figure 2, lower panel), the signal at m/z 147 decreases by almost 2 orders of magnitude. This is due to the dissociation of the precursor by collisions with the plasma species. Since the signals obtained by mass spectrometry result from electron-impact dissociative ionization in the ionization chamber, the signal at $\mathrm{m} / \mathrm{z} 147$ is considered to be exclusively originating from HMDSO (m/z 162) entering the $\mathrm{PSMS}^{25}$. Using the values at plasma-off and on states, a dissociation degree of HMDSO ( $\tau_{\mathrm{HMDSO}}$ ) of about $95 \pm 5 \%$ is obtained; it follows the formula: $\tau_{\mathrm{HMDSO}}(\%)=\left(1-147_{\text {on }} / 147_{\text {off }}\right) \times 100$. This corroborates the previous results presented by Despax et al. ${ }^{37}$ obtained in the same plasma reactor and with the same applied power. It further denotes a strong reproducibility of the measurements. Similarly to $\mathrm{m} / \mathrm{z} 147$, other peaks 
related to species originating from HMDSO dissociation undergo a decrease, though less severe. This implies possibilities of new schema of formation for the latter after plasma ignition i.e., their formation path might not follow only the dissociative ionization of the HMDSO precursor. Peaks above m/z 162 are usually observed when fragment ions interact with the HMDSO molecule and create bigger compounds through oligomerization ${ }^{30}$. Such signals were not detected in significant amounts under the present operation conditions, most likely due to the small quantity of injected HMDSO. Note that, by PSMS, we did not measure features related to the Ag species; this would be ascribed to the low sputtering yield of the metallic species due to low applied rf power combined with the target poisoning. Another reason might be the location of the PSMS probe head which is positioned at a port-hole on the reactor wall.

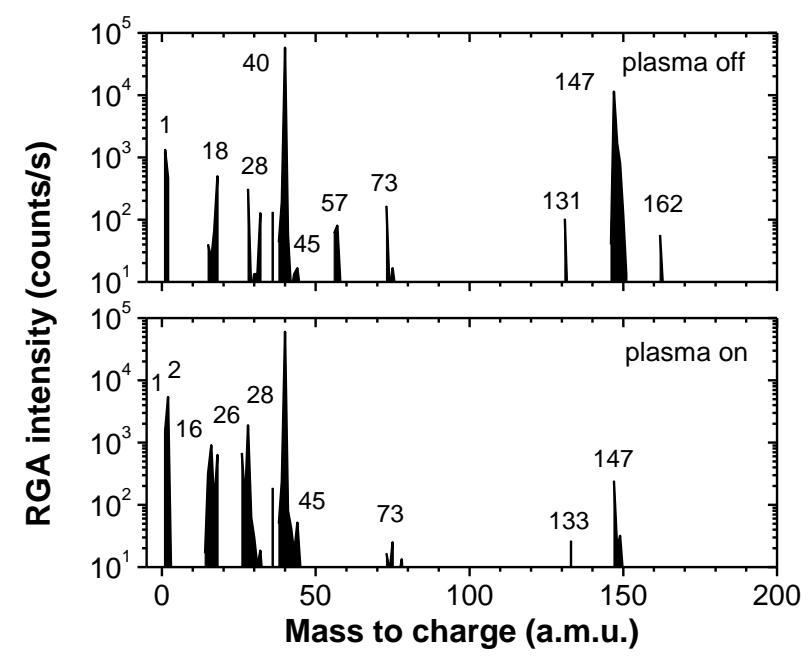

Figure 2: Mass spectra obtained with PSMS at plasma on and off. Conditions: power $=30 \mathrm{~W}, \mathrm{Ar}$ base pressure $=40$ mTorr, Ar flow $=2.8 \mathrm{sccm}$, average HMDSO flow $=0.12 \mathrm{sccm} \&$ duty cycle $=30 \%(1.5 \mathrm{~s} / 5 \mathrm{~s})$.

Besides the strong decrease of the HMDSO-related signals observed after plasma ignition, new features also appear with the plasma-on, as reported previously ${ }^{37}$. The two peaks at 
$\mathrm{m} / \mathrm{z} 16$ and 26 are attributed to methane $\left(\mathrm{CH}_{4}\right)$ and acetylene $\left(\mathrm{C}_{2} \mathrm{H}_{2}\right)$, respectively. These species cannot be created by simple or multiple dissociations of HMDSO. However, the plasma induces fragmentation that leads to the creation of many products having particularly strong signals at plasma-on state, notably methyl radicals, atomic hydrogen and molecular hydrogen, at m/z 2 . Generally $\mathrm{H}_{2}$ cannot be formed as a product of the precursor dissociation; it may only be created by recombination either in the gas phase (negligible at low pressure) or by surface reactions on the electrodes, on plasma reactor walls, or on nanoparticles (e.g. Eley-Ridea ${ }^{45}$ ). Moreover, small hydrocarbon molecules $\left(\mathrm{C}_{\mathrm{x}} \mathrm{H}_{\mathrm{y}}, \mathrm{x}, \mathrm{y}=1,2\right)$ may be formed by recombination reactions involving methyl radicals ${ }^{46}$ as well as through surface reactions with carbonaceous materials ${ }^{47}$. The signal at $\mathrm{m} / \mathrm{z} 133$ has been previously attributed to $\mathrm{Si}_{2} \mathrm{OH}\left(\mathrm{CH}_{3}\right)_{4}{ }^{+}$, but a signal is also observed at $\mathrm{m} / \mathrm{z} 131$ at plasma-off, then attributed to $\mathrm{Si}_{2} \mathrm{OC}_{4} \mathrm{H}_{11}{ }^{+37}$. Such result could be explained if the former comes from the recombination of the latter with atomic hydrogen in the gas phase. It should be noted that the signal at $\mathrm{m} / \mathrm{z} 73$ can also be linked to a fragment involving recombination with atomic hydrogen, $\mathrm{Si}_{2} \mathrm{OH}^{+}$, but is usually in negligible proportion compared to the $\mathrm{Si}\left(\mathrm{CH}_{3}\right)_{3}{ }^{+}$reported here ${ }^{25,30}$.

The dynamics induced by the pulsed injection of HMDSO at plasma-off state is presented in Figure 3 which depicts the evolution of the main HMDSO-related signals, identified in Figure 2, over 20 injection cycles with an HMDSO duty cycle of 70\% (3.5 s / $5 \mathrm{~s}$ ). In order to highlight the oscillations of each feature, the recorded spectra are normalized to their maximum value. An offset is also applied to improve clarity in the representation. Only the temporal variations of $\mathrm{Si}_{2} \mathrm{O}\left(\mathrm{CH}_{3}\right)_{5}{ }^{+}$at $\mathrm{m} / \mathrm{z} 147$ are noticeable, though very light, and enable indirect observation of the injection pulses. The rest of the signals present a lot of noise since they are very weak at plasmaoff state (see Figure 2). The small amplitude of the oscillations of $\mathrm{Si}_{2} \mathrm{O}\left(\mathrm{CH}_{3}\right)_{5}{ }^{+}$implies that 
HMDSO has a long residence time either in the plasma reactor or in the plasma sampling mass spectrometer chamber as compared with the period of the pulses.

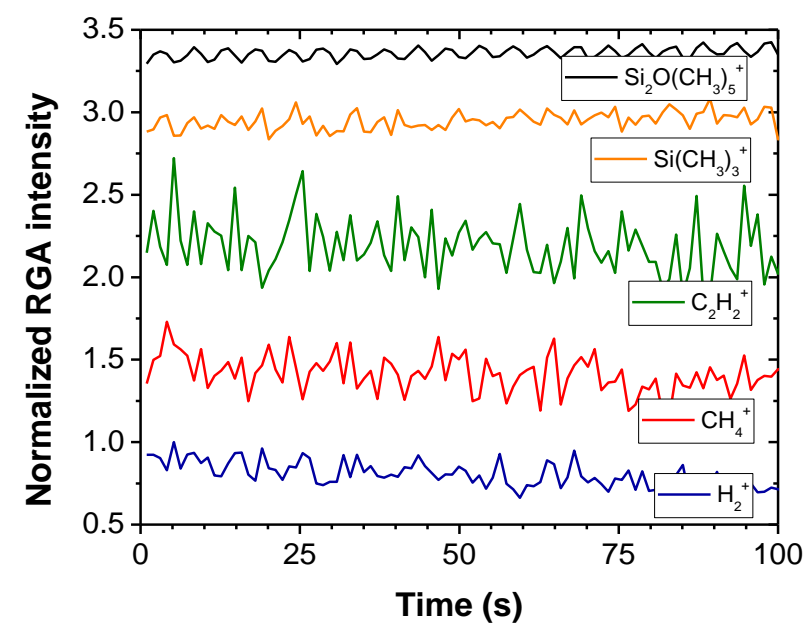

Figure 3: Temporal evolution of Residual Gas Analysis (RGA) intensity of the main masses obtained by PSMS at plasma off. For clarity, the signals were normalized and offset on the vertical axis. Conditions: power $=10 \mathrm{~W}$ (not applied for plasma off), Ar base pressure $=40$ mTorr, Ar flow $=2.8 \mathrm{sccm}$, average HMDSO flow $=0.28 \mathrm{sccm}$ and duty cycle $=70 \%(3.5 \mathrm{~s} / 5 \mathrm{~s})$. These are the standard conditions and will remain fixed for the rest of the study.

Figure 4 presents similar measurements as in Figure 3, but at plasma-on state. The rf power was fixed to $10 \mathrm{~W}$. This power was found to be particularly suitable for successive formation and disappearance of $\mathrm{dust}^{36}$. The peaks observed at plasma-on state, except the one at m/z 147 (corresponding to HMDSO entering the PSMS ionization chamber), are corrected by the contribution of the cracking pattern of HMDSO. This contribution can be determined from the signals with the plasma-on and -off states by using $\left(X=X_{\text {on }}-X_{\text {off }} *\left(147_{\text {on }} / 147_{\text {off }}\right)\right)$. Here, the $147_{\text {on }} / 147_{\text {off }}$ ratio takes into account the change in the HMDSO population by fragmentation upon discharge ignition. $\mathrm{H}_{2}{ }^{+}, \mathrm{CH}_{4}{ }^{+}$and $\mathrm{C}_{2} \mathrm{H}_{2}{ }^{+}$present different behaviour compared to that of the sizable fragments derived from HMDSO dissociation. Indeed, while the fragments at m/z 73 and 
147 (corresponding to $\mathrm{Si}\left(\mathrm{CH}_{3}\right)_{3}{ }^{+}$and $\mathrm{Si}_{2} \mathrm{O}\left(\mathrm{CH}_{3}\right)_{5}{ }^{+}$, respectively) strongly vary with the injection pulses, the oscillations on the 5-s time-scale of the small hydrocarbons formed in the plasma reactor is reduced, implying that they are not as much dependent on the immediate introduction of HMDSO. Regarding $\mathrm{H}_{2}$, it may be formed in the gas phase when $\mathrm{H}$ atoms recombine in threebody reactions, but this process is not favoured in our low-pressure conditions. It is indeed verified by the complete absence of any variations of $\mathrm{H}_{2}{ }^{+}$signal on the 5-s time-scale. It can therefore be concluded that $\mathrm{H}_{2}$ is solely formed by surface reactions. In this framework, weak oscillations of $\mathrm{CH}_{4}{ }^{+}$and especially $\mathrm{C}_{2} \mathrm{H}_{2}{ }^{+}$features also suggest that surface reactions are the main formation pathway for these hydrocarbons; we will come back to this point later on.

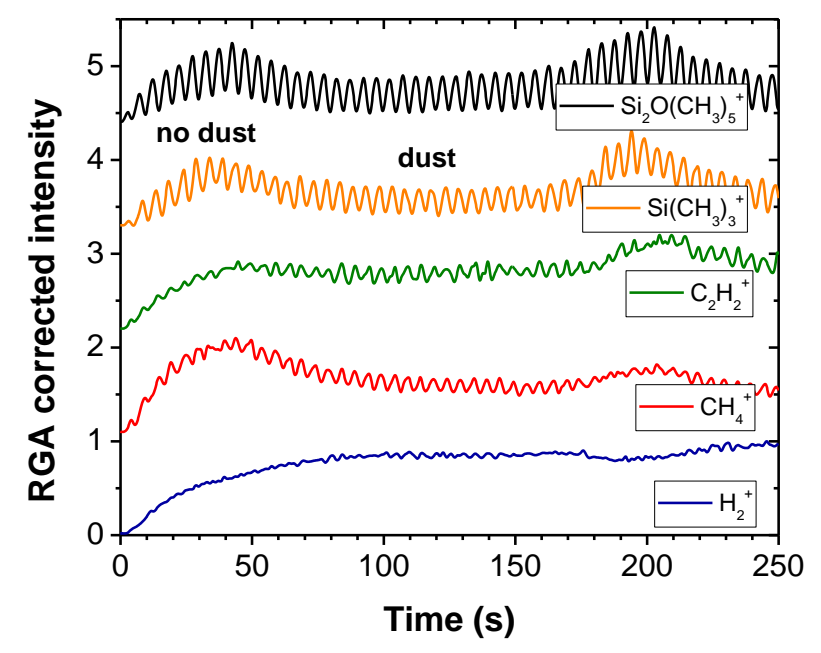

Figure 4: Temporal evolution of RGA intensity of the principal masses obtained by PSMS with the plasma on. Intensities are normalized, and an offset is applied to improve clarity. Standard conditions.

The effect of dust formation on the population of HMDSO and its fragments is also important, as revealed by the dynamics occurring on a time-scale of a few hundred seconds. In this experiment, $\mathrm{t}=0 \mathrm{~s}$ is set as the beginning of HMDSO addition in the nominally pure argon 
plasma. After $50 \mathrm{~s}$, the signature of dust formation starts being observable on the PSMS measurements. Most of the signals decrease in dusty conditions, and increase back at the end of the dust cycle, close to $\mathrm{t}=200 \mathrm{~s}$. However, $\mathrm{H}_{2}{ }^{+}$does not follow this trend, and even slightly increases with dust formation. Different explanations may be advanced on this observation. On one hand, the presence of dust can increase the formation of $\mathrm{H}_{2}$ by Eley-Rideal reactions on the surface of the nanoparticles. On the other hand, the presence of dust is known to increase the electron temperature which could influence the dissociation degree of HMDSO in the plasma, thus resulting in a rise of atomic and possibly molecular hydrogen ${ }^{43,48}$. However, since dust formation follows a cyclic behaviour, it implies that they grow simultaneously until a critical size is reached, leading to their loss by gravity ${ }^{42}$. If collection of plasma species by the dust was significant, a continuous decrease in the signals of HMDSO-related fragments would be noticeable in dusty conditions. Such a decrease is not observed in Figure 4. This indicates that surface reactions on nanoparticles play a negligible role in our conditions, implying that the increase of $\mathrm{H}_{2}$ is related to a rise of the dissociation degree; this must inevitably yield to a rise in the population of $\mathrm{H}$ atoms and therefore to the population of $\mathrm{H}_{2}$ by recombination on the electrodes and plasma reactor walls. A purely technical reason for the accumulation of $\mathrm{H}_{2}$ can however be related to difficulties in the $\mathrm{H}_{2}$ pumping, and certainly contributes to the continuous increase observed in Figure 4. To verify that the increase of $\mathrm{H}_{2}$ is also induced by the dissociation degree and to investigate the precursor fragmentation kinetics, OES have been coupled with PSMS measurement. 


\section{Populations of HMDSO-related species over multiple time-scales}

\subsection{Dissociation of the parent molecule}

After observation of different dynamical phenomena regarding the HMDSO dissociation kinetics, the results depicted in Figure 3 and Figure 4 have been used to calculate the signal ratio $147_{\text {off }} / 147_{\text {on }}$. This value is an indicator of the plasma induced dissociation of the parent molecule; it cannot be lower than 1 (no fragmentation) and will rise when the HMDSO becomes dissociated by interaction with plasma species. Temporal evolution of the $147_{\text {off }} / 147_{\text {on }}$ ratio is shown in Figure 5. Experiments carried out with a continuous HMDSO injection yielded a dissociation degree of $95 \pm 5 \%$ using the $\mathrm{m} / \mathrm{z} 147$ signals (HMDSO-15) at plasma-on and -off states. In such case, no dust growth occurred. However, when HMDSO is introduced by pulses, cyclic variations on the recorded signals corresponding to both the pulsed injection of HMDSO and the formation and disappearance of the dust cloud are observed. The addition of HMDSO through pulses $\left(\mathrm{t}_{\mathrm{on}} / \mathrm{T}=3.5 \mathrm{~s} / 5 \mathrm{~s}\right)$ tends to lower the dissociation of the precursor in the plasma, while the growth of nanoparticles increases the HMDSO-dissociation. As mentioned above, OES measurements of $\operatorname{Ar}$ Ar 2p-to-1s transitions in the 700-900 nm wavelength range were recorded simultaneously with PSMS. They were coupled with the predictions of a collisional-radiative modelling describing the population of emitting $\operatorname{Ar} 2 p$ states to obtain the evolution of the electron temperature (assuming Maxwellian electron energy distribution function) and electron density. More details on this method can be found in our previous works ${ }^{43,48}$. These quantities are then used as input parameters to investigate the complex precursor fragmentation kinetics over multiple time-scales, namely the pulsed injection of HMDSO and the formation of dust.

This was first realized for the HMDSO parent molecule using a simple particle balance equation (eq. (1)). 


$$
\frac{\partial n_{H M D S O}}{\partial t}+\nabla \cdot \Gamma=G_{0}(t)-k_{\text {dissociation }}\left(T_{e}\right) n_{e} n_{H M D S O}
$$

where $n_{H M D S O}$ and $n_{e}$ are the precursor and electron densities, respectively. Generally, inertia effects are important at very low pressure, where description of a free-fall regime is aimed at. Given the working pressure and the large mass of HMDSO, transport effects, represented by the divergence of the flux, $\Gamma$ can be neglected. The assumption on the HMDSO-diffusion is confirmed and presented at the end of the section. In this case, it is assumed that the precursor is injected into the gas phase through a rate $G_{0}(t)$ and is lost by electron-impact dissociation. Other dissociation channels, like dissociation induced by interactions with Ar metastable atoms and UV photons are neglected ${ }^{33}$. Charge transfer reactions between Ar ions and precursor molecules that were reported in other studies at pressures ranging between 0.4 and 300 Torr are also disregarded since, in the current conditions, the ion-molecule interactions are limited by the low number density and low kinetic energy of the ions ${ }^{30,32,34}$. As previously mentioned, surface reactions with nanoparticles may also be neglected for both creation (through reformation of the molecule) and loss of HMDSO. Since the phenomena related to the population and depopulation of HMDSO occur at rates significantly greater than the one at which OES and PSMS measurements were recorded, a quasi-steady state can be assumed at each measurement time $t$. In equation (1), $k_{\text {dissociation }}\left(T_{e}\right)$ is the dissociation rate of $\mathrm{HMDSO}$, mostly towards $\mathrm{Si}_{2} \mathrm{O}\left(\mathrm{CH}_{3}\right)_{5}$, as evidenced by the HMDSO cracking pattern in Figure 2. In addition, as ascribed in Hess et al. ${ }^{49}$, this reaction is characterized by a much larger cross section over the other dissociation pathways of HMDSO. This reaction thus corresponds to the electron-impact breaking of a bond between a methyl group and a silicon atom (bond dissociation energy of $3.7 \mathrm{eV}^{49}$ ). For simplicity, Thompson's model ${ }^{50}$ for inelastic collisions is used to express the dissociation rate of HMDSO as a function of the electron temperature $T_{e}$ and the bond dissociation energy $E$ : 


$$
k_{\text {dissociation }}\left[\mathrm{cm}^{3} / \mathrm{s}\right]=\pi\left(\frac{e}{4 \pi \varepsilon_{0} E}\right)^{2} \sqrt{\frac{8 e T_{e}}{\pi m_{e}}}\left(1+\frac{2 T_{e}}{E}\right) \exp \left(\frac{-E}{T_{e}}\right) .
$$

Equations (1) and (2) were used to calculate the time-dependant frequency related to the dissociation of HMDSO:

$$
\frac{G_{0}}{n_{H M D S O}}=v_{\text {diss }}=k_{\text {dissociation }}\left(T_{e}\right) n_{e}
$$

where $v_{\text {diss }}=k_{\text {dissociation }}\left(T_{e}\right) n_{e}$ is calculated using the time-resolved values of the electron temperature and electron number density obtained from OES measurements ${ }^{43,48}$. The main interest in this quantity obtained uniquely from OES data is that it can directly be compared with PSMS data. More precisely, $G_{0}$, relates to the sudden injection of HMDSO, which can be considered proportional to the peak at $\mathrm{m} / \mathrm{z} 147$ with the plasma off, whereas the number density of HMDSO, $n_{H M D S O}$ is proportional to the intensity of the same peak at plasma on. $G_{0} / n_{H M D S O}$ is therefore directly proportional to the signal ratio $147_{\text {off }} / 147_{\text {on }}$. Both sets of data are presented in Figure 5. The top graph presents the evolution of the dissociation frequency obtained by OES using equation (2) on a $5 \mathrm{~s}$ time-scale. It appears that the latter decreases during the on-time of the HMDSO injection pulses, often by a factor of two or more. In previous studies ${ }^{43,48}$, HMDSO was found to strongly influence the electron population of the plasma, leading to an immediate decrease in electron temperature and a sudden rise in the electron number density. The oscillations observed in Figure 5 are nonetheless mostly linked to variations of the electron temperature $^{43}$ (recall that the dissociation rate increases exponentially with $T_{e}$ in Thompson's $\operatorname{model}^{50}$ ). The electron temperature is also responsible for the variation of the dissociation frequency over a few hundred seconds. Indeed, when the dust growing cycle initiates, newlyformed particles collect free electrons; a process that depends on their size and density ${ }^{51,52}$. The addition of a loss mechanism for free electrons in dusty conditions results in an increase in 
electron temperature ${ }^{43,48}$, which in turn increases the dissociation frequency of HMDSO, as observed in Figure 5.

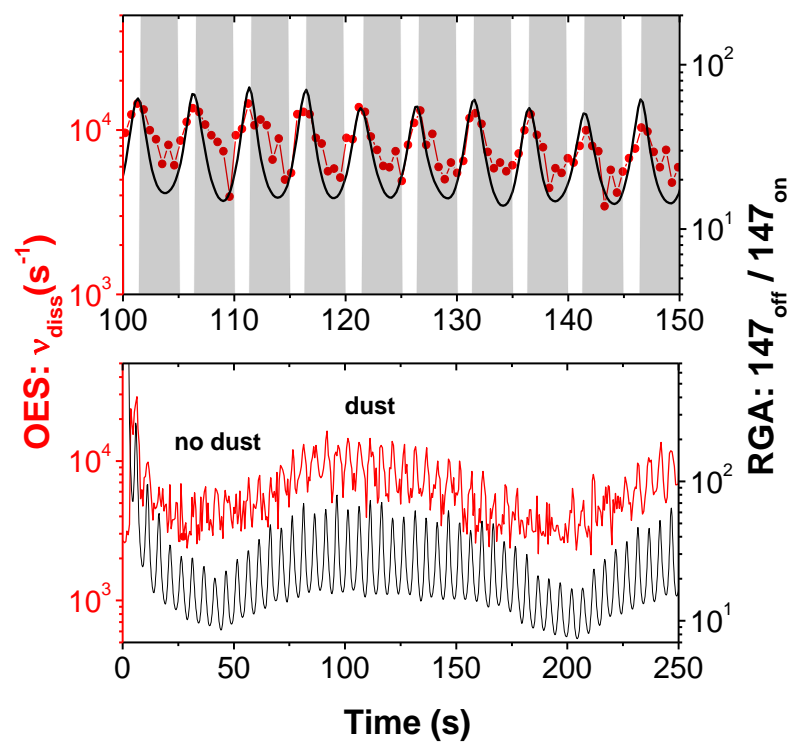

Figure 5: Temporal development of the dissociation frequency (left vertical scale) and of the $\mathrm{m} / \mathrm{z} 147$ ratio at plasma off and on (right). Time during the HMDSO injection pulses are represented as grey areas in the top graph. Standard conditions.

Figure 5 further reveals that the temporal evolution of the dissociation degree obtained exclusively by OES and equation (3) matches perfectly the one determined solely by PSMS from the $147_{\text {off }} / 147_{\text {on }}$ ratio. More precisely, they decrease similarly qualitatively during all HMDSO injection cycles. Both quantities also increase threefold with the dust growth. The fact that the $147_{\text {off }} / 147_{\text {on }}$ ratio remains between 10 and 100 means that the dissociation degree of HMDSO varies between 91 and $99 \%$, peaking in dusty conditions. These results also confirm our assumptions; the gain of HMDSO is related to its injection (recombination between fragments for the parent molecule reformation can be neglected) and the primary loss mechanism of HMDSO is electron impact dissociation. Hence, diffusion effects of the precursor and/or surface 
reactions involving the electrodes, the reactor walls or the nanoparticles (in dusty conditions) are not significantly contributing to losses over the range of conditions examined.

In order to examine the possible contribution of diffusion effects, the framework presented in Liebermann et al. ${ }^{50}$ was used to treat the HMDSO-diffusion in the $\nabla \cdot \Gamma$ term in equation (1). Assuming a diffusion coefficient of $0.08 \mathrm{~m}^{2} / \mathrm{s}$ in the $40 \mathrm{mTorr}$, Ar/HMDSO plasma, and partially adsorbing reactor walls characterized by a sticking coefficient $\gamma^{53}$, the $G_{0} / n_{H M D S O}$ ratio can be expressed as :

$$
\frac{G_{0}}{n_{H M D S O}}=v_{\text {diss }}\left[1+\frac{\sqrt{D}}{v_{\text {diss }}^{1 / 2} l}\left(\operatorname{coth}\left(l \sqrt{\frac{D}{v_{\text {diss }}}}\right)+\frac{2(2-\gamma)}{\gamma} \sqrt{D v_{\text {diss }} \frac{\pi m_{H M D S O}}{8 k T}}\right)^{-1}\right]^{-1},
$$

where $l$ is the inter-electrode distance, $D$ is the diffusion coefficient, $T$ is the temperature of HMDSO (assumed at $300 \mathrm{~K}$ ) and $v_{\text {diss }}$ represents the electron-impact dissociation frequency. In equation (4), the term in the brackets is linked to diffusion effects and thus to the reactor dimensions and surface reaction coefficient. Figure 6 shows the predictions of equation (4) for various values of $\gamma$. The results show that the dissociation frequency would be slightly affected when the sticking coefficient reaches 1 - which would be an important overestimation in the case of the fully coordinated HMDSO precursor ${ }^{54}$. Variations with $\gamma$ further indicate a quick asymptotic behaviour towards what is obtained if diffusion effects are neglected. Based on these findings, it can be concluded that electron-impact dissociation is the most relevant pathway for the loss of the HMDSO parent molecule, which amplifies the importance of the electron kinetics in this kind of hybrid PVD/PECVD processes. 


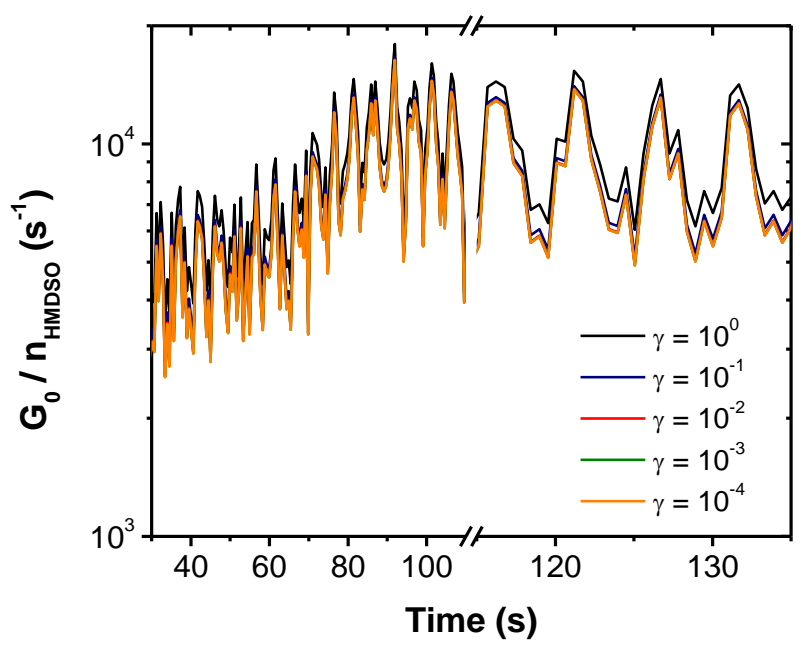

Figure 6: Effect of the sticking coefficient of HMDSO parent molecule on the chamber walls on the calculated $G_{0} / n_{H M D S O}$ ratio. Standard conditions.

\subsection{Creation and losses of HMDSO fragments}

From the results presented above, HMDSO dissociation by electron impact can produce a number of fragments, including $\mathrm{Si}_{2} \mathrm{O}\left(\mathrm{CH}_{3}\right)_{5}$. These fragments can be further dissociated, for example by the loss of another methyl group by electron impact ${ }^{27}$. Recombination reactions between fragments (mostly on the electrodes and reactor walls due to the low pressure) can also produce other organic and organosilicon species. The creation and loss dynamics of each of these species in Ar/HMDSO plasmas is obviously complex and most likely vary from one fragment to another. For these reasons, it is not possible to establish a simple particles balance equation for all HMDSO fragments. However, dissociation by electron impact certainly plays key role in both their creation and loss dynamics. In this regard, it is worth highlighting that a change in the dissociation kinetics due to variations of the electron temperature would not yield the same result as those linked to variations of the electron number density. If only the electron number density varies, the dissociation degree should follow, but it seems unclear how the relative densities of 
the fragments would behave ${ }^{27}$. On the other hand, if the electron temperature changes, the occurrence of the various fragmentation reactions will vary according to their energy-dependent cross sections and thus the $T_{e}$-dependant reaction rates. For example, in the case of a fragment $\mathrm{X}$ that is created by electron-impact dissociation of HMDSO (with a production rate given by $k_{\text {diss }}\left(T_{e}\right) n_{e} n_{H M D S O}$ ) and lost through subsequent dissociation (with a loss rate given by $\left.k_{\text {diss }}^{\prime}\left(T_{e}\right) n_{e} n_{X}\right)$, the particle balance equation, under steady-state conditions with the dissociation rates expressed in the form of equation (2), can be expressed as :

$$
\frac{n_{x}}{n_{\text {HMDSO }}} \propto \exp \left(\frac{-\Delta E}{T_{e}}\right),
$$

where $\Delta E=E-E$ ' represents the difference between the two bond dissociation energies (the electron-impact dissociation of HMDSO leading to a fragment $\mathrm{X}$ and the electron-impact dissociation of the fragment $\mathrm{X}$ leading to a smaller fragment $\mathrm{Y}$ ). HMDSO is a sizable and thus complex molecule (as compared to those involved in more commonly used plasma deposition processes), with each bond having its own specific energy. The $\mathrm{Si}-\mathrm{O}$ bond is the strongest one in HMDSO (dissociation energy of 6-9 eV) while the Si-C is the weakest one, ranging around 3.5$6 \mathrm{eV}^{49,55}$. The bond dissociation energy depends on the number of atoms involved in the molecule. A good example is the case of the $\mathrm{C}-\mathrm{H}$ bond in a $\mathrm{CH}_{\mathrm{x}}$ group: $\mathrm{CH}_{4} \rightarrow \mathrm{CH}_{3}+\mathrm{H}$ takes $4.55 \mathrm{eV}$, but $\mathrm{CH}_{3} \rightarrow \mathrm{CH}_{2}+\mathrm{H}$ takes $4.77 \mathrm{eV}, \mathrm{CH}_{2} \rightarrow \mathrm{CH}+\mathrm{H}$ takes $4.38 \mathrm{eV}$ and $\mathrm{CH} \rightarrow \mathrm{C}+\mathrm{H}$ takes $3.5 \mathrm{eV}^{56}$. It is however considered that the bond dissociation energy decreases with the bulkiness of the molecule ${ }^{49}$. Depending on the fragment and the corresponding dissociation process involved, $\Delta E$ could therefore be either positive or negative. Hence, the number density of the fragment, with respect to the population of HMDSO, should either increase or decrease with a change of the electron temperature. If the energy difference is positive, an increase in $T_{e}$ would 
lead to an increase in the fragment-to-precursor number density ratio. Over the range of experimental conditions investigated, with $T_{e}$ variations of the order of $0.15 \mathrm{eV}$ over the precursor injection cycle and $0.3 \mathrm{eV}$ over the formation and disappearance of the dust cloud, $\Delta E$ values of at least a few eVs are required to observe significant changes in the population ratio.

It was shown in Figure 4 that signals related to HMDSO varied differently with the pulsed injection of the precursor and, at a much lower frequency, with the growth of nanoparticles in the gas phase. Specifically, most signals decrease in dusty conditions compared to non-dusty conditions. We previously assigned this feature to a change in the dissociation degree of HMDSO following an increase in the electron temperature with the formation of nanoparticles, as shown in Figure 5. It is however not clear how this phenomenon affects the fragmentation pattern of HMDSO, from the $\mathrm{Si}_{2} \mathrm{O}\left(\mathrm{CH}_{3}\right)_{5}$ fragment to the other species.

To gain more insight into the fragmentation path of HMDSO and its multi-scale behaviour, the signals were first smoothed to filter out the variations over $5 \mathrm{~s}$. Results are shown in Figure 7. Firstly, the ratio of $\mathrm{m} / \mathrm{z} 73$ over $\mathrm{m} / \mathrm{z} 147$ present no significant variation. Since $T_{e}$ significantly varies over this $250 \mathrm{~s}$ time-scale, the fragment related to the peak at $\mathrm{m} / \mathrm{z} 73$ most likely has comparable bond energies involved in the electron-impact dissociation of HMDSO with respect to the one involved in the electron-impact dissociation of the fragment. The former case could happen if both dissociations induce a loss of one methyl group. On the other hand, PSMS ratios of the fragments found at $\mathrm{m} / \mathrm{z} 2,16$ and 26 become dominant in dusty conditions where the electron temperature reaches a maximum. It should also be noted that, contrary to the peak at $\mathrm{m} / \mathrm{z} 73$, these three species are not observed in measurements realized with the plasma off (see Figure 2) and are thus uniquely formed in the plasma following multiple reactions involving not only electron-impact dissociation but also recombination on the plasma-exposed 
surfaces. In such cases, it appears difficult to analyze the results in the context of equation (5) in which both production and loss rates are based on electron-impact dissociation reactions.

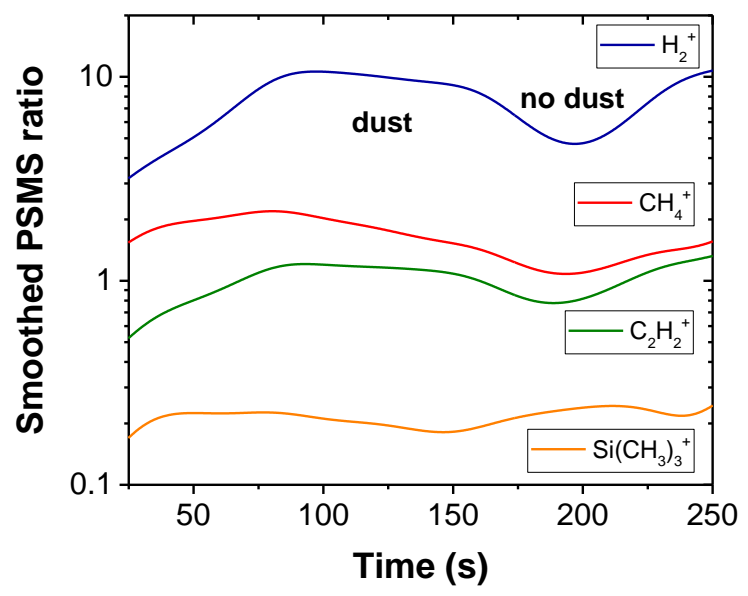

Figure 7: Temporal variation of the fragment-to-parent molecule PSMS ratios $(\mathrm{m} / \mathrm{z} 2$ $133 / \mathrm{m} / 2$ 147) during the formation and disappearance of the dust cloud. Standard conditions.

Previous studies realized in methane plasmas in contact with carbonaceous surfaces have revealed that part of the methane and most of the acetylene are created by surface recombination reactions between atomic hydrogen and small hydrocarbon compounds deposited on the electrodes and plasma reactor walls ${ }^{45-47,57,58}$. In this context, assuming that acetylene is uniquely populated through heterogeneous surface reactions and lost by electron impact dissociation, its density $n_{\mathrm{C}_{2} \mathrm{H}_{2}}$ can be expressed as:

$$
n_{C_{2} H_{2}} \propto \frac{[H]_{s, g}\left[C_{x} H_{y}\right]_{s}}{k_{d i s s}\left(T_{e}\right) n_{e}},
$$

where $\left[\mathrm{C}_{\mathrm{x}} \mathrm{H}_{\mathrm{y}}\right]_{s}$ represents the population of hydrocarbon species on the plasma-exposed surfaces and $[\mathrm{H}]_{s, g}$ corresponds to either the gas phase population of $\mathrm{H}$ atoms in the case of Eley-Rideal recombination or the population of $\mathrm{H}$ atoms adsorbed on the plasma-exposed surfaces in the case 
of Langmuir-Hinshelwood recombination. Evidently, atomic hydrogen in the plasma is linked to HMDSO dissociation, $\mathrm{H}$ being easily dissociated from every fragment of the precursor $(\mathrm{H}$ is the most abundant species injected in the gas phase with 18 atoms per parent molecule). As discussed above, typical energies involved in $\mathrm{H}$ formation from the electron-impact dissociation of hydrocarbon and organosilicon compounds are between 3.5 and $4.77 \mathrm{eV}^{56}$. On the other hand, acetylene can be dissociated by electron-impact fragmentation of the $\mathrm{C}-\mathrm{H}$ bond with a higher threshold of $5.77 \mathrm{eV}$ (the triple $\mathrm{C}-\mathrm{C}$ bond at $10 \mathrm{eV}$ is generally considered unaltered) ${ }^{38}$. Therefore, the relative population of acetylene with respect to HMDSO, as depicted by the $\mathrm{m} / \mathrm{z}$ 26-to-m/z 147 PSMS ratio, should follow the trend of the electron temperature, in very good agreement with the results presented in Figure 7. A similar framework can probably be established for the other species in which surface recombination reactions play an important role, in particular $\mathrm{H}_{2}$ and methane.

Figure 8 presents the fragment-to-parent molecule PSMS ratios over the time-scale of the HMDSO injection. Again, as in Figure 7, no significant change is observed for the $\mathrm{m} / \mathrm{z} 73$-to$\mathrm{m} / \mathrm{z} 147$ ratio. This confirms that the bond energy involved in the electron-impact dissociation of HMDSO leading to $\mathrm{Si}\left(\mathrm{CH}_{3}\right)_{3}$ is comparable to the bond energy involved in the electron-impact dissociation of the fragment (both dissociations involved a loss of one methyl group). On the other hand, it appears that the plasma tends towards a more $\mathrm{H}_{2-}$, methane- and acetylene-rich discharge during the HMDSO injection off time. Again, the variations observed over this timescale are due to the same phenomenon as the one observed during the formation and loss of nanoparticles. In the context of equation (6), the acetylene-to-HMDSO PSMS ratio should first decrease with the corresponding diminution of the electron temperature following HMDSO injection. These ratios should then increase in the HMDSO injection off-time with the 
subsequent rise of $T_{e}$. These trends are consistent with those observed in the experiment. It is worth highlighting that the acetylene-to-HMDSO PSMS ratio varies by about a factor of 1.5 for a variation of $T_{e}$ by a factor of 1.2 between dusty and non-dusty plasma conditions. On the other hand, this ratio varies by about a factor of 4 for a variation of $T_{e}$ by a factor of 1.1 during the HMDSO injection cycles. The more prominent variations observed with HMDSO injection for similar variations of $T_{e}$ is therefore most likely ascribed to a change in the surface coverage by hydrocarbon species (linked to the quantities $\left[\mathrm{C}_{\mathrm{x}} \mathrm{H}_{\mathrm{y}}\right]_{s}$ in equation (6)).

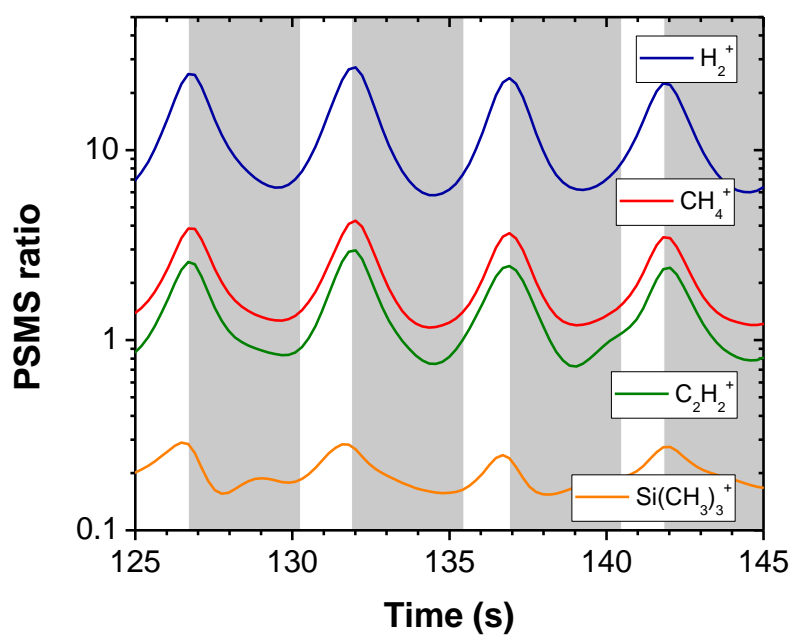

Figure 8: Temporal variation of the fragment/monomer ratios $(\mathrm{m} / \mathrm{z} 2-133 / \mathrm{m} / \mathrm{z}$ 147) during pulsed injection of HMDSO. HMDSO injection pulses are represented by grey areas. Standard conditions.

Formation of acetylene and subsequent electron attachment reactions have been found to lead to dust formation in hydrocarbon plasmas, notably by forming the $\mathrm{C}_{2} \mathrm{H}^{-}$anion. This also includes discharges with methane as a precursor ${ }^{38}$. Following the observations obtained over multiple time scales, it seems that the pulsed injection of HMDSO would contribute to dust 
nucleation by enhancing the populations of these well-known dust promoters (acetylenic compounds) during the off-time of the HMDSO injection cycles.

\section{Conclusion}

In this work, the time-resolved fragmentation kinetics of HMDSO has been examined in a low-pressure argon rf discharge with conditions promoting dust growth. Multi-scale oscillations of the HMDSO-related signals obtained by plasma sampling mass spectroscopy in the residual gas analysis mode were found: this includes the pulsed injection of the precursor (time-scale: $5 \mathrm{~s}$ ) and the formation and disappearance of the dust cloud (time-scale: $150 \mathrm{~s}$ ). OES measurements of Ar 2p-to-1s transitions were coupled with the predictions of a collisionalradiative model describing the populations of emitting Ar $2 p$ states to determine the timedependent values of the electron temperature and electron number density. These values were then used as input parameters in a particle balance equation for the parent molecule to obtain an electron-impact dissociation frequency. This quantity obtained solely from OES measurements was compared to the $147_{\text {off }} / 147_{\text {on }}$ ratio obtained by PSMS. An excellent qualitive agreement was reached over both time scales. More precisely, the dissociation degree respectively increases and decreases with dust growth and HMDSO injection pulses. These results confirm our assumptions that losses of HMDSO on the electrodes, reactor walls and nanoparticles (in dusty conditions) are negligible with respect to its electron-impact dissociation.

Comparing non-dusty and dusty conditions, small hydrocarbons, which are considered to play an important role in the formation of radicals and thus in the nucleation of nanoparticles, were found in greater densities than the precursor. Such results imply that their consumption while forming dust is not the leading loss factor of these species. Moreover, compounds 
generated by volume and surface recombination between atomic hydrogen and HMDSO fragments or carbonaceous species contained within the hydrocarbon deposit were found to be favorized in these conditions. This is especially true for $\mathrm{H}_{2}, \mathrm{CH}_{4}$ and $\mathrm{C}_{2} \mathrm{H}_{2}$ that are mainly formed through surface reactions with the deposit on the electrodes and plasma reactor walls. Besides, our results give insights into the effect of a variation of the electron temperature regarding the fragmentation pattern of HMDSO. This is of significant interest since it can considerably improve the plasma process control. Indeed, the change in plasma chemistry due to the presence of dust particles would induce unwanted inhomogeneities in the composition of the deposit. By plotting fragment-to-monomer ratios, it was found that the plasma tends towards a methane and acetylene-rich discharge rather than an HMDSO one. Since acetylene is a wellknown dust promoter, this means that the pulsed injection of HMDSO in such conditions enhances the dust growth.

\section{Acknowledgements}

This work was supported by the Natural Sciences and Engineering Research Council of Canada through both the Discovery Grants Program and the Postgraduate Scholarships-Doctoral Program. The authors also acknowledge support from the European Research Council under the European Union's Seventh Framework Program ERC-2013-SyG, Grant Agreement n. 610256 NANOCOSMOS. This work has also been supported by the CNRS-UdeM network GDRI "Nanomatériaux Multifonctionnels Contrôlés - NMC". 


\section{References}

${ }^{1}$ F. Massines, N. Gherardi, A. Fornelli, and S. Martin, Surf. Coatings Technol. 200, 1855 (2005).

${ }^{2}$ L. Zajickova, V. Bursikova, Z. Kucerova, D. Franta, P. Dvorak, R. Smid, V. Perina, and A. Mackova, Plasma Sources Sci. Technol. 16, S123 (2007).

${ }^{3}$ D.S. Wavhal, J. Zhang, M.L. Steen, and E.R. Fisher, Plasma Process. Polym. 3, 276 (2006).

${ }^{4}$ L. Martinu and D. Poitras, J. Vac. Sci. Technol. A Vacuum, Surfaces, Film. 18, 2619 (2000).

${ }^{5}$ S.E. Alexandrov and M.L. Hitchman, Chem. Vap. Depos. 11, 457 (2005).

${ }^{6}$ M. Moravej and R.F. Hicks, Chem. Vap. Depos. 11, 469 (2005).

${ }^{7}$ D. Trunec, L. Zajickova, V. Bursikova, V. Studnicka, P. Stahel, V. Prysiazhnyi, V. Perina, J. Houdkova, Z. Navratil, and D. Franta, J. Phys. D. Appl. Phys. 43, 225403 (2010).

${ }^{8}$ R. Jafari, S. Asadollahi, and M. Farzaneh, Plasma Chem. Plasma Process. 33, 177 (2013).

${ }^{9}$ L. Xie, Z. Tang, L. Jiang, V. Breedveld, and D.W. Hess, Surf. Coatings Technol. 281, 125 (2015).

${ }^{10}$ O. Levasseur, L. Stafford, N. Gherardi, N. Naudé, V. Blanchard, P. Blanchet, B. Riedl, and A. Sarkissian, Plasma Process. Polym. 9, 1168 (2012).

${ }^{11}$ B.C. Trasferetti, R. V Gelamo, F.P. Rouxinol, and M.A. Bica de Moraes, Chem. Mater. 16, 567 (2004).

${ }^{12}$ B. Despax and P. Raynaud, Plasma Process. Polym. 4, 127 (2007).

${ }^{13}$ C. Saulou, B. Despax, P. Raynaud, S. Zanna, P. Marcus, and M. Mercier-Bonin, Appl. Surf. Sci. 256, S35 (2009).

${ }^{14}$ L. Milliere, K. Makasheva, C. Laurent, B. Despax, and G. Teyssedre, Appl. Phys. Lett. 105, (2014).

${ }^{15}$ H. Wang, J. Lin, and Z.X. Shen, J. Sci. Adv. Mater. Devices 1, 225 (2016). 
${ }^{16}$ F. Hilt, N. Gherardi, D. Duday, A. Berné, and P. Choquet, ACS Appl. Mater. Interfaces 8, 12422 (2016).

${ }^{17}$ K. Maex, M.R. Baklanov, D. Shamiryan, F. Iacopi, S.H. Brongersma, and Z.S. Yanovitskaya, J. Appl. Phys. 93, 8793 (2003).

${ }^{18}$ R.P. Patel and C.A. Wolden, J. Vac. Sci. Technol. A 29, 0210121 (2011).

${ }^{19}$ D. Hegemann, H. Brunner, and C. Oehr, Surf. Coatings Technol. 142-144, 849 (2001).

${ }^{20}$ H. Caquineau, I. Enache, N. Gherardi, N. Naudé, and F. Massines, J. Phys. D. Appl. Phys. 42, 125201 (2009).

${ }^{21}$ A. Granier, M. Vervloet, K. Aumaille, and C. Vall e, Plasma Sources Sci. Technol. 12, 89 (2003).

${ }^{22}$ M. Goujon, T. Belmonte, and G. Henrion, Surf. Coatings Technol. 188-189, 756 (2004).

${ }^{23}$ K. Aumaille, C. Vallée, A. Granier, A. Goullet, F. Gaboriau, and G. Turban, Thin Solid Films 359, 188 (2000).

${ }^{24}$ A.S.C. Nave, F. Mitschker, P. Awakowicz, and J. Röpcke, J. Phys. D. Appl. Phys. 49, 1 (2016).

${ }^{25}$ R. Basner, R. Foest, M. Schmidt, K. Becker, and H. Deutsch, Int. J. Mass Spectrom. 176, 245 (1998).

${ }^{26}$ M.R. Alexander, F.J. Jones, and R.D. Short, J. Phys. Chem. B 101, 3614 (1997).

${ }^{27}$ M.R. Alexander, F.R. Jones, and R.D. Short, Plasmas Polym. 2, 277 (1997).

${ }^{28}$ J. Benedikt, D. Ellerweg, S. Schneider, K. Rügner, R. Reuter, H. Kersten, and T. Benter, J. Phys. D. Appl. Phys. 46, (2013).

${ }^{29}$ V. Rouessac, S. Roualdes, and J. Durand, Chem. Vap. Depos. 8, 155 (2002).

${ }^{30}$ C.Q. Jiao, C.A. DeJoseph, and A. Garscadden, J. Vac. Sci. Technol. A 23, 1295 (2005). 
${ }^{31}$ K. Li, O. Gabriel, and J. Meichsner, J. Phys. D. Appl. Phys. 37, 588 (2004).

${ }^{32}$ M. Creatore, Y. Barrell, J. Benedikt, and M.C.M. Van De Sanden, Plasma Sources Sci.

Technol. 15, 421 (2006).

${ }^{33}$ J.L. Jauberteau and I. Jauberteau, J. Phys. Chem. A 116, 8840 (2012).

${ }^{34}$ S. Carles, J.L. Le Garrec, and J.B.A. Mitchell, J. Chem. Phys. 127, 1 (2007).

${ }^{35}$ C. Laurent and E. Kay, J. Appl. Phys. 65, 1717 (1989).

${ }^{36}$ B. Despax, K. Makasheva, and H. Caquineau, J. Appl. Phys. 112, 093302 (2012).

${ }^{37}$ B. Despax, F. Gaboriau, H. Caquineau, and K. Makasheva, AIP Adv. 6, 105111 (2016).

${ }^{38}$ C. Deschenaux, A. Affolter, D. Magni, C. Hollenstein, and P. Fayet, J. Phys. D. Appl. Phys.

32, 1876 (1999).

${ }^{39}$ K. De Bleecker, A. Bogaerts, and W. Goedheer, Phys. Rev. E 73, 026405 (2006).

${ }^{40}$ M. Calafat, D. Escaich, R. Clergereaux, P. Raynaud, and Y. Segui, Appl. Phys. Lett. 91, 181502 (2007).

${ }^{41}$ J. Winter, J. Berndt, S.-H. Hong, E. Kovačević, I. Stefanović, and O. Stepanović, Plasma Sources Sci. Technol. 18, 034010 (2009).

${ }^{42}$ J. Berndt, E. Kovačević, I. Stefanović, O. Stepanović, S.H. Hong, L. Boufendi, and J. Winter, Contrib. to Plasma Phys. 49, 107 (2009).

${ }^{43}$ V. Garofano, R. Bérard, S. Boivin, C. Joblin, K. Makasheva, and L. Stafford, to appear in Plasma Sources Sci. Technol. (2019).

${ }^{44}$ J.E. Drake, B.M. Glavinčevski, and C. Wong, J. Inorg. Nucl. Chem. 42, 175 (1980).

${ }^{45}$ M. Wittmann and J. Küppers, J. Nucl. Mater. 227, 186 (1996).

${ }^{46}$ D. Magni, C. Deschenaux, C. Hollenstein, A. Creatore, and P. Fayet, J. Phys. D. Appl. Phys. 34, 87 (2001). 
${ }^{47}$ A. von Keudell, C. Hopf, W. Jacob, and A. Von Keudell, Nucl. Fusion 39, 1451 (1999).

${ }^{48}$ V. Garofano, L. Stafford, B. Despax, R. Clergereaux, and K. Makasheva, Appl. Phys. Lett. 107, 183104 (2015).

${ }^{49}$ G.G. Hess, F.W. Lampe, and L.H. Sommer, J. Am. Chem. Soc. 87, 5327 (1965).

${ }^{50}$ M.A. Lieberman and A.J. Lichtenberg, Principle of Plasma Discharges and Materials

Processing (Jonh Wiley \& Sons, Inc., 2005).

${ }^{51}$ T. Fukuzawa, M. Shiratani, and Y. Watanabe, Appl. Phys. Lett. 64, 3098 (1994).

${ }^{52}$ Y. Watanabe, J. Phys. D. Appl. Phys. 39, R329 (2006).

${ }^{53}$ P. Chantry, J. Appl. Phys. 62, 1141 (1987).

${ }^{54}$ A. Michelmore, P.M. Bryant, D.A. Steele, K. Vasilev, J.W. Bradley, and R.D. Short, Langmuir 27, 11943 (2011).

${ }^{55}$ N.E. Blanchard, B. Hanselmann, J. Drosten, M. Heuberger, and D. Hegemann, Plasma Process. Polym. 12, 32 (2015).

${ }^{56}$ S.J. Blanksby and G.B. Ellison, Acc. Chem. Res. 36, 255 (2003).

${ }^{57}$ S. Stoykov, C. Eggs, and U. Kortshagen, Inst. Phys. Publ. 2160, (2001).

${ }^{58}$ K.M. Ervin, J. Ho, and W.C. Lineberger, J. Chem. Phys. 91, 5974 (1989). 